\title{
Contributos para a Validação da Versão Portuguesa do EQ-5D
}

\author{
Contribution for the Validation of the Portuguese Version of EQ-5D
}

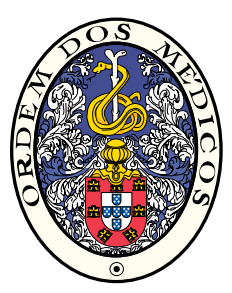

\author{
Pedro Lopes FERREIRA ${ }^{1,3}$, Lara Noronha FERREIRA ${ }^{2,3}$, Luis Nobre PEREIRA L $^{2,4}$ \\ Acta Med Port 2013 Nov-Dec;26(6):664-675
}

\section{RESUMO}

Introdução: O EQ-5D permite a junção de duas componentes essenciais de qualquer medida de qualidade de vida relacionada com a saúde a ser usada em avaliações económicas de custo-utilidade: (i) um perfil descrevendo o estado de saúde em termos de domínios ou dimensões; e (ii) um valor numérico associado ao estado de saúde anteriormente descrito.

Objectivo: A versão portuguesa do questionário EQ-5D foi finalizada em 1998, com base em normas de orientação definidas pelo Grupo EuroQol, incluindo os procedimentos de tradução e retroversão. Apesar da sua larga utilização em Portugal, até agora ainda não tinham sido publicados os estudos que conduziram inicialmente à versão portuguesa e à garantia de aceitabilidade, fiabilidade e validade. O propósito do presente artigo é, assim, documentar estes primeiros valores referentes à versão portuguesa do EQ-5D.

Material e Métodos: Foram utilizadas três amostras diferentes: uma primeira com 1500 indivíduos representativa da população portuguesa; uma segunda com 140 indivíduos apenas destinada ao teste da fiabilidade; e uma terceira amostra com 643 indivíduos doentes com cataratas, asma, doença pulmonar obstrutiva crónica ou artrite reumatoide.

Resultados: A aceitabilidade foi avaliada pelo número de respostas em falta. Foi também encontrado um marcado efeito de teto com grande parte da amostra a não reportar quaisquer problemas nas dimensões do EQ-5D.

Discussão: A validade de construção foi testada pela análise do grau com que valores baixos de EQ-5D estavam positivamente associados ao aumento da idade, ao ser do sexo feminino, e ao estar doente, assim como a valores de dimensões da escala SF-36v2. A validade convergente foi baseada nas correlações entre valores do EQ-5D e outras escalas específicas de condição de saúde. 0 EQ-5D apresentou correlações moderadas a altas com outras medidas de estado de saúde e de qualidade de vida relacionada com a saúde, específicas de cada doença.

Conclusão: A versão portuguesa do EQ-5D tem uma boa aceitabilidade, fiabilidade e validade na medição do estado de saúde.

Palavras-chave: Estado de Saúde; Portugal; Qualidade de Vida; Medição de Saúde; Questionários.

\section{ABSTRACT}

Introduction: The EQ-5D allows the achievement of two essential components of any measure of health-related quality of life to be used in cost-utility economic evaluations: (i) a profile describing the health status in terms of domains or dimensions; and (ii) a numeric value associated with the health status described.

Aim: The Portuguese version of the EQ-5D questionnaire was completed in 1998, based on guidelines set by the EuroQol Group, including translation and back translation procedures. Despite its wide use in Portugal, until now it had not yet been published studies that initially led to the Portuguese version and the guarantee of acceptability, reliability and validity. The purpose of this article is to document these first values relating to the Portuguese version of the EQ-5D.

Material and Methods: We used three different samples: a first one with 1,500 individuals representative of the Portuguese population; a second with 140 individuals just intended for the reliability test; and a third sample with 643 individual patients with cataracts, asthma, chronic obstructive pulmonary disease, or rheumatoid arthritis.

Results: The acceptability was assessed by the number of missing responses. It was also found a marked ceiling effect, with a large part of the sample not reporting any problems in the dimensions of the EQ-5D.

Discussion: The construct validity was tested by examining the degree to which low values of EQ-5D were positively associated with increasing age, being female, and the sick, as well as the values of dimensions of the SF-36v2 scale. Convergent validity was based on correlations between EQ-5D values and other specific measures. The EQ-5D showed moderate to high correlations with other diseasespecific measures of health status and health related quality of life.

Conclusion: We can state that the Portuguese version of the EQ-5D has a good accessibility, reliability and validity in measuring health.

Keywords: Health Status; Health Surveys; Quality of Life; Portugal; Questionnaires.

\section{INTRODUÇÃO}

\section{Enquadramento}

$O$ EQ-5D é um instrumento genérico de medição da qualidade de vida relacionada com a saúde (QdVRS) que permite gerar um índice representando o valor do estado de saúde de um indivíduo. Desenvolvido pelo grupo
EuroQoL a partir de $1987^{1}$ e tornado público desde $1990,^{2}$ é baseado num sistema classificativo que descreve a saúde em cinco dimensões: mobilidade, cuidados pessoais, atividades habituais, dor/mal-estar e ansiedade/depressão. Cada uma destas dimensões tem três níveis de gravidade

1. Faculdade de Economia. Universidade de Coimbra. Coimbra. Portugal.

2. Escola Superior de Gestão, Hotelaria e Turismo. Universidade do Algarve. Faro. Portugal.

3. Centro de Estudos e Investigação em Saúde da Universidade de Coimbra. Coimbra. Portugal.

4. Centro de Investigação sobre o Espaço e as Organizações. Universidade do Algarve. Faro. Portugal.

Recebido: 27 de Janeiro de 2013 - Aceite: 13 de Agosto de 2013 | Copyright @ Ordem dos Médicos 2013 
associados, correspondendo a sem problemas (nível 1), alguns problemas (nível 2) e problemas extremos (nível 3) vividos ou sentidos pelo indivíduo. Assim sendo, este sistema permite descrever um total de $3^{5}=243$ estados de saúde distintos.

A principal razão para o desenvolvimento inicial deste instrumento de medição é que, antes dele, havia apenas questionários, como o SF-36 e o NHP, que permitem obter um perfil de saúde e serem utilizados em avaliações económicas de custo-efetividade. Estes, no entanto, não permitem o cálculo de índices genéricos cardinais que representem o valor (intensidade de preferências) atribuído ao estado de saúde e que possam ser utilizados em avaliações económicas de custo-utilidade. Foi, assim, intenção do grupo EuroQoL a criação de um índice genérico cardinal de saúde para aplicação em avaliações económicas. Pretenderam também criar um instrumento de medição que completasse as restantes medidas e que viabilizasse a recolha de dados de referência em comparações multinacionais.

Segundo os seus autores, o sistema descritivo foi obtido após três fases que incluíram uma primeira revisão dos principais instrumentos de medição genéricos de estado de saúde então existentes, uma análise resultante da experiência da aplicação destes instrumentos pelos membros do grupo e a evidência obtida em entrevistas face-a-face. ${ }^{1}$ Para além do preenchimento deste sistema descritivo é pedido ao respondente que registe a avaliação que faz do seu estado de saúde em geral numa escala visual analógica de 0 (pior estado de saúde imaginável) a 100 (melhor estado de saúde imaginável) denominada frequentemente por termómetro EQ-VAS.

O EQ-5D é um instrumento de medição de autopreenchimento. A descrição do estado de saúde do respondente, conseguida através do sistema classificativo composto pelas cinco escalas com valores de 1 a 3 , e o termómetro EQ-VAS são as duas componentes mais vulgarmente utilizadas pelos investigadores e prestadores de cuidados apenas interessados na obtenção de informação sobre o impacto do estado de saúde na vida e na qualidade de vida dos indivíduos. No entanto, as respostas a este sistema descritivo podem também ser agregadas através de um algoritmo sensível aos valores da sociedade, isto é, aos valores que os indivíduos associam a cada um dos estados de saúde, produzindo um índice de valor.

\section{Sistema descritivo do EQ-5D}

A Tabela 1 apresenta as dimensões e os níveis sistema descritivo do EQ-5D.

Para cada indivíduo, o resultado desta descrição é representado através de um número de cinco dígitos. Assim, por exemplo, o estado 21132 corresponde ao estado de saúde de uma pessoa com alguns problemas em andar, sem problemas em cuidar de si e em desempenhar as suas atividades habituais, com dores ou mal-estar extremos e moderadamente ansiosa ou deprimida.

Para além desta descrição, e para garantir uma primeira aproximação dos ganhos em saúde, em especial quando se trata de uma primeira avaliação, o EQ-5D permite ainda que o respondente forneça, comparando com o seu nível geral de saúde nos 12 meses anteriores, uma perceção do seu estado de saúde. Nesta comparação, é pedido que escolha entre as opções de resposta 'melhor', 'o mesmo' e 'pior'.

\section{Valor associado ao estado de saúde}

O EQ-5D pressupõe duas formas de associar valor a um estado de saúde de uma pessoa. A primeira, a completar a descrição do estado de saúde, oferece ao respondente a possibilidade de localizar o seu próprio estado de saúde numa escala visual analógica como a representada na figura 1. Utilizando a técnica de medição direta, ${ }^{3}$ é solicitado ao respondente que trace uma linha entre a 'caixa' que representa o seu estado de saúde nesse momento e o

Tabela 1 - Sistema descritivo do EQ-5D.

Dimensão Nível

(1) Não tenho problemas em andar

Mobilidade

(2) Tenho alguns problemas em andar

(3) Tenho de estar na cama

Cuidados pessoais

(1) Não tenho problemas em cuidar de mim

(2) Tenho alguns problemas a lavar-me ou vestir-me

(3) Sou incapaz de me lavar ou vestir sozinho/a

Atividades habituais

Dor/mal-estar

Ansiedade/depressão
(1) Não tenho problemas em desempenhar as minhas atividades habituais

(2) Tenho alguns problemas em desempenhar as minhas atividades habituais

(3) Sou incapaz de desempenhar as minhas atividades habituais

(1) Não tenho dores ou mal-estar

(2) Tenho dores ou mal-estar moderados

(3) Tenho dores ou mal-estar extremos

(1) Não estou ansioso/a ou deprimido/a

(2) Estou moderadamente ansioso/a ou deprimido/a

(3) Estou extremamente ansioso/a ou deprimido/a 
termómetro EQ-VAS de 0 a 100 , considerando 0 o pior estado de saúde imaginável e 100 o melhor estado de saúde imaginável (Fig.1).

No entanto, é de realçar que esta escala visual analógica, apesar de ser um processo simples de atribuir valor, não permite representar o compromisso entre quantidade e qualidade de vida ${ }^{4}$ e, por essa razão, não fornece valores cardinais ou utilidades e não pode contribuir com pesos para a determinação de QALY (quality-adjusted life years).

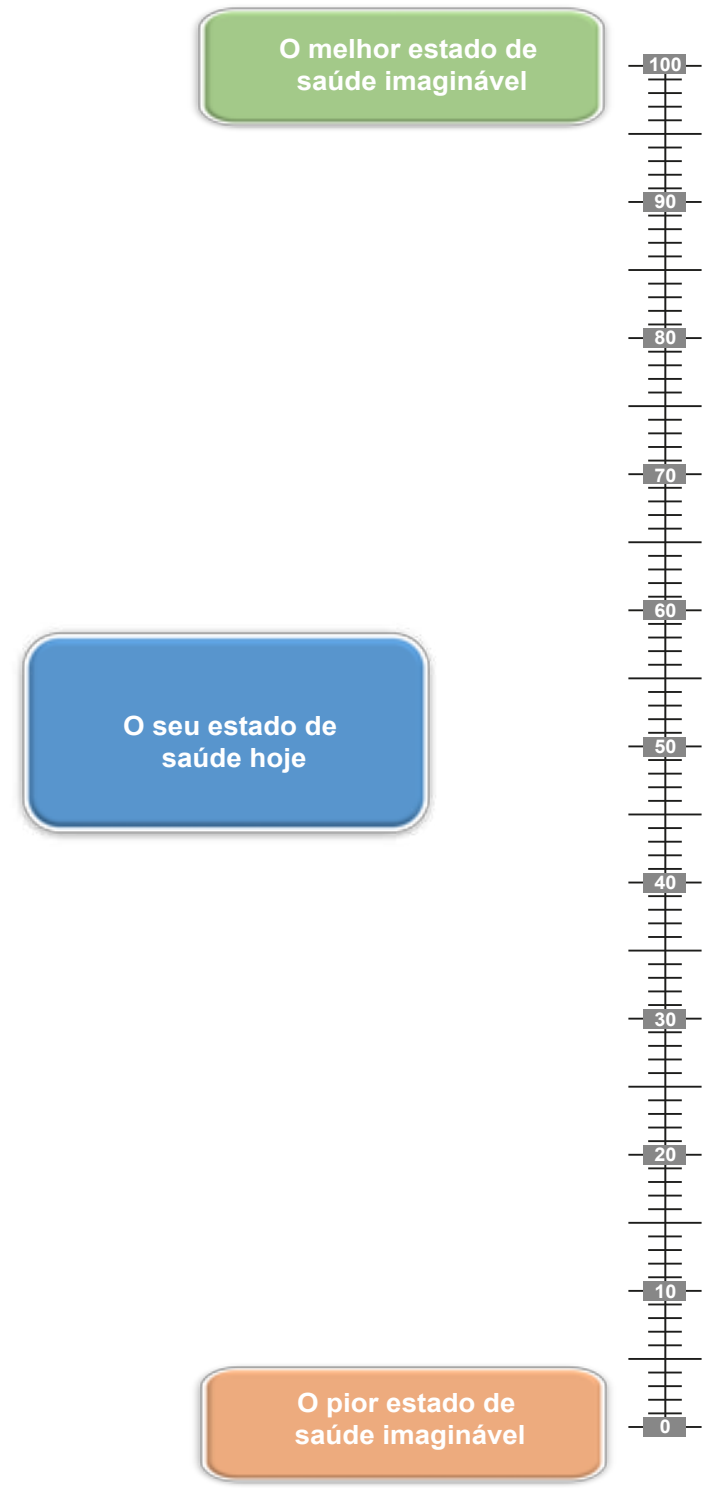

Figura 1 - Termómetro EQ-VAS.

Tabela 2 - Sistema descritivo do EQ-5D. ${ }^{5}$
A segunda forma de fazer associar um valor a um estado de saúde específico, após a sua descrição e classificação, é obter um valor com base nas preferências recolhidas da população em geral. Este valor situa-se numa escala de 1 (saúde perfeita) a 0 (morte), admitindo, contudo, valores negativos correspondentes a estados de saúde considerados como piores do que morte.

A abordagem inicial para determinar este valor de preferência foi desenvolvida por Dolan et $\mathrm{a}^{5,6} \mathrm{com}$ base num conjunto inicial de 45 estados de saúde e 2997 membros da população britânica, selecionados num estudo denominado MVH (medição e valoração da saúde) levado a cabo pela Universidade de York. ${ }^{7}$

Através da aplicação da técnica de regressão generalizada dos mínimos quadrados, estes investigadores encontraram uma função aditiva que permite, para o contexto britânico, transformar um número de cinco dígitos resultante da descrição de um estado de saúde num índice cardinal que varia entre -0,59 e 1,00. Este índice é sensível ao nível da gravidade de cada dimensão, inclui um termo constante a para qualquer estado de saúde diferente de 11111 e um termo N3 identificador de um nível mais grave de qualquer dimensão. Isto é, a função aditiva é definida por

$$
\mathrm{V}=1-\alpha-\mathrm{MO}-\mathrm{CP}-\mathrm{AH}-\mathrm{DM}-\mathrm{AD}-\mathrm{N} 3
$$

em que 1 representa o valor da saúde perfeita utilizado para reescalar o resultado final no intervalo de 0 a 1 , a é uma constante que representa a não coincidência do valor de qualquer dimensão em relação ao nível 1, N3 é uma outra constante que representa a presença do nível 3 em qualquer dimensão e as variáveis $\mathrm{MO}, \mathrm{CP}, \mathrm{AH}, \mathrm{DM}$ e $A D$ assumem os valores fornecidos pelas primeiras cinco linhas da Tabela 2 para o respetivo nível de cada uma das cinco dimensões.

Por exemplo, para o estado de saúde 21132 atrás referido encontramos um valor de índice obtido da seguinte forma:

$V=1-0,081-0,069-0,000-0,000-0,386-0,071-0,269=0,124$

Com um índice determinado deste modo é possível obter o valor de QALY associado a este estado de saúde, ${ }^{8}$ i.e., fornecer uma medida de benefício dos cuidados de saúde que possa ser usada em avaliações económicas.

Vários são os países europeus e não europeus com sistemas de valores adaptados ao próprio país e/ou normas para o EQ-5D (veja-se, por exemplo).,9-13 Em Portugal, com o apoio financeiro do Alto Comissariado da Saúde e dos

\begin{tabular}{llcc}
\hline Dimensão & Nível 1 & Nível 2 & Nível 3 \\
\hline (MO) Mobilidade & 0,000 & 0,069 & 0,314 \\
(CP) Cuidados pessoais & 0,000 & 0,104 & 0,214 \\
(AH) Atividades habituais & 0,000 & 0,036 & 0,094 \\
(DM) Dor/mal-estar & 0,000 & 0,123 & 0,386 \\
(AD) Ansiedade/depressão & 0,000 & 0,071 & 0,236 \\
& $\alpha$ & & 0,081 \\
& N3 & & \\
\end{tabular}


Laboratórios Pfizer, o Centro de Estudos e Investigação em Saúde da Universidade de Coimbra (CEISUC) conduziu um grande projeto de medição e valoração da saúde através do $E Q-5 D$ com as primeiras publicações já disponíveis online. ${ }^{14,15}$ Dividido em duas partes (valoração e normas) teve como objetivos (1) validar para a realidade portuguesa o EQ-5D, (2) determinar um sistema de valores para Portugal adaptado à realidade cultural portuguesa e (3) medir a QdVRS da população portuguesa, através da aplicação do EQ-5D e, assim, determinar as respetivas normas portuguesas.

O objetivo do presente artigo é documentar os primeiros valores referentes à versão portuguesa do EQ-5D. Começaremos, assim, pelo processo seguido de criação da versão portuguesa do EQ-5D e da sua equivalência semântica e linguística, após o que analisaremos aspetos da sua validação.

\section{MATERIAL E MÉTODOS \\ Dados}

Para este artigo, recolhemos dados essencialmente provenientes de três amostras:

Na primeira amostra, os dados foram recolhido por uma empresa de sondagem especialmente contratada para o efeito através de entrevistas telefónicas realizadas a uma amostra aleatória estratificada por NUTS II, género e idade de 1500 indivíduos da população portuguesa com, pelo menos, 18 anos de idade e residente em todas as regiões de Portugal (18 distritos do continente, Açores e Madeira). Os investigadores só tiveram acesso aos dados anonimizados.

Os 150 registos da segunda amostra foram também recolhidos pela mesma empresa de sondagem, tendo cada indivíduo participado numa entrevista telefónica com um espaço de uma semana de intervalo. Nesta amostra, embora os entrevistadores da empresa conhecessem obviamente a identidade das pessoas que tinham de entrevistar de novo, nunca essa informação foi colocada na base de dados nem revelada aos investigadores.

A terceira amostra com 643 indivíduos foi composta por doentes distribuídos por quatro patologias: 352 tinham cataratas, 115 tinham asma, 72 tinham doença pulmonar obstrutiva crónica (DPOC) e 104 tinham artrite reumatoide (AR). Os asmáticos e os doentes com DPOC foram recrutados por pneumologistas de uma forma sequencial durante pouco mais de um ano. ${ }^{16}$ Os doentes com cataratas foram selecionados de entre os que estavam em lista de espera para cirurgia em dois hospitais públicos ${ }^{17}$ e os doentes com AR estavam registados numa base de dados da Liga Portuguesa de Combate às Doenças Reumáticas (LPCDR) e foram referenciados por reumatologistas. ${ }^{18}$

Todas estas amostras tiveram o consentimento explícito e informado dos doentes, para além da autorização das respetivas Comissões de Ética. Os doentes com dificuldade de preenchimento, por doença ou por iliteracia, foram ajudados por profissionais de enfermagem.

\section{Análise estatística}

Aceitabilidade. Para testar esta propriedade pretendeu-se verificar a exequibilidade do EQ-5D e até que ponto é bem aceite pelos respondentes. Com base nas três amostras, foram testadas as taxas de resposta e as taxas de instrumentos completamente preenchidos. Partiu-se das seguintes hipóteses:

$\mathrm{H}_{1}$ : Os indivíduos não apresentam grandes problemas no preenchimento do $E Q-5 D$, o que se reflete numa baixa taxa de dados omissos;

$\mathrm{H}_{2}$ : A EQ-VAS, sendo um pouco mais complexa do que o sistema descritivo, estará associada a uma percentagem maior de dados omissos.

Distribuição. Os dados das três amostras permitiram a análise da distribuição das respostas. Pretendeu-se também identificar a existência de efeito de teto, i.e., de grande percentagem de indivíduos reportando valores elevados de estado de saúde. Partiu-se, assim, da seguinte hipótese:

$\mathrm{H}_{3}$ : A parte descritiva do EQ-5D apresenta efeito de teto.

Fiabilidade. Como o EQ-5D é essencialmente utilizado em estudos de avaliação económica e de políticas de saúde e não na prática clínica, a fiabilidade foi testada a nível agregado. A fiabilidade teste-reteste foi avaliada na amostra 2 em que cada indivíduo foi interrogado duas vezes num período de duas semanas. Utilizaram-se, para isso, o coeficiente $\mathrm{k}$ de Cohen e os critérios definidos por Landis e Koch. ${ }^{19}$ Por outro lado, a coerência interna foi medida através do indicador $\alpha$ de Cronbach e com os critérios de qualificação definidos. ${ }^{20}$

Validade de construção. Para testar este tipo de validade foi feita uma análise das correlações existentes entre as pontuações do EQ-5D na amostra 1 e, na amostra 3, em condições específicas de saúde. Ambas as amostras permitiram também relacionar os valores obtidos pelo EQ-5D ou pela EQ-VAS com as variáveis sociodemográficas ou com valores do SF-36v2, um instrumento que permite medir oito principais dimensões em saúde, todas elas através de vários itens e de uma escala de 0 a 100, com os extremos a corresponderem, respetivamente, à pior e à melhor QdVRS possíveis. As dimensões medidas são a função física (FF), as limitações de desempenho devido a problemas físicos (DF) ou emocionais (DE), a intensidade e o desconforto causado pela dor (DR), a saúde em geral (SG), a vitalidade (VT), a função social (FS) e a saúde mental (SM). ${ }^{21-24}$

Foram testadas as seguintes quatro hipóteses para o EQ-5D e outras quatro para o EQ-VAS:

$\mathrm{H}_{4}$ : Indivíduos que indiquem problemas em qualquer dimensão do EQ-5D terão valores mais baixos em todas as escalas do SF-36v2;

$\mathrm{H}_{5}$ : Indivíduos referenciados com problemas na dimensão ansiedade/depressão do EQ-5D apresentarão maiores reduções nas dimensões FS e SM do SF-36v2;

$\mathrm{H}_{6}$ : Indivíduos com problemas de dor/mal-estar reportam também menores valores na dimensão DR do SF$-36 v 2$;

$\mathrm{H}_{7}$ : Indivíduos mais idosos ou que declaram ter uma 

do de saúde;

doença apresentam piores valores nas dimensões de esta-

$\mathrm{H}_{8}$ : Valores de EQ-VAS serão mais altos em indivíduos que refiram ter melhor saúde na respetiva escala de autoavaliação do SF-36v2;

$\mathrm{H}_{9}$ : Os valores de EQ-VAS correlacionam-se negativamente com o aumento de idade;

$\mathrm{H}_{10}$ : Indivíduos que declaram ter uma doença apresentam valores mais baixos de EQ-VAS;

$\mathrm{H}_{11}$ : As mulheres reportam valores de EQ-VAS mais baixos do que os homens.

Estas hipóteses foram testadas usando os testes do qui-quadrado, $t$ de Student, de Mann-Whitney e de Kruskall-Walis e o coeficiente de correlação de Spearman. O método de Scheffé foi usado para o ajuste a comparações múltiplas. Por outro lado, testaram-se modelos de regressão múltipla para verificar a relação entre o EQ-5D e os valores obtidos pelos instrumentos específicos de condição. Para testar a validade discriminativa verificou-se se os três níveis de resposta de cada dimensão discriminavam ou não a incapacidade funcional medida pelo SF-36v2.

Validade de critério. Na inexistência de um 'padrão-ouro' para a medição da QdVRS, a validade de critério foi testada por intermédio da comparação com os valores de utilidade obtidos pelo SF-6D na amostra 1 e com os dados obtidos na amostra 3 recolhida em doentes com patologias e que, simultaneamente, preencheram medidas específicas da respetiva patologia.

O SF-6D é outro índice baseado em preferências que resulta da conversão de 11 itens do SF-36 num sistema de classificação com seis dimensões, cada uma com quatro a seis níveis, permitindo a geração de um total de 18000 estados de saúde diferentes. ${ }^{25,26} \mathrm{O}$ sistema descritivo do SF-6D é composto pelas seguintes dimensões: função física, limitação de desempenho, função social, dor, saúde mental e vitalidade.

Assim, para as subamostras referentes às cataratas, à asma, à $D P O C$ e à artrite reumatoide, para além do EQ-5D e do SF-6D, foram utilizadas as versões portuguesas do Catquest, do Asthma Quality of Life Questionnaire (AQLQ(S)), do Asthma Control Questionnaire (ACQ), do Clinical COPD Questionnaire (CCQ) e do Arthritis Impact Measurement Scales 2 (AIMS2-SF).

O Catquest foi desenhado com o objetivo de analisar os resultados da cirurgia às cataratas, não só no respeitante à acuidade visual, mas também aos impactos da cirurgia nas atividades diárias, nas limitações visuais, nos sintomas relacionados com as cataratas e no grau de independência dos doentes. ${ }^{27,28}$

O AQLQ é um questionário específico para doentes com asma com perguntas relativas a quatro domínios (sintomas, limitações da atividade, função emocional e exposição ambiental). O AQLQ(S) é uma versão padronizada do $\mathrm{AQLQ}^{29,30}$ em que as cinco perguntas do domínio das atividades são genéricas e correspondem às cinco atividades mais escolhidas pelos doentes na versão AQLQ original.

$\mathrm{O} A C Q$ é um questionário pequeno composto por sete itens e também desenvolvido por Juniper et $\mathrm{al}^{31}$ com o objetivo de medir o controlo da asma. Cinco destes itens dizem respeito aos sintomas e às limitações da atividade, um à percentagem de FEV1 prevista e outro à utilização de broncodilatadores de curta duração.

O CCQ é um pequeno questionário clínico que mede o estado de saúde de doentes com dificuldades respiratórias devido à DPOC, permitindo obter informação sobre a gravidade dos sintomas, as limitações por eles provocadas e o efeito do tratamento. ${ }^{32}$ Está disponível em várias línguas e em duas versões: uma semanal e outra de 24 horas que pode ser usada como um diário. É composto por dez itens, divididos em três domínios: sintomas, estado funcional e estado mental.

Finalmente, o AIMS2-SF é um instrumento específico de medição da qualidade de vida de doentes com artrite reumatoide inicialmente desenvolvido por Meenan e colegas $^{33}$ e sujeito a várias revisões e alterações. ${ }^{34}$ Permite a medição de cinco componentes de qualidade de vida: física, sintomas, afeto, interação social e desempenho.

Esta forma de validade convergente foi testada através das seguintes hipóteses e da utilização dos coeficientes de correlação de Speaman e de Pearson:

$\mathrm{H}_{12}$ : Correlação entre EQ-5D/VAS e SF-6D;

$\mathrm{H}_{13}$ : Correlação entre EQ-5D/VAS e Catquest;

$\mathrm{H}_{14}$ : Correlação entre EQ-5D/VAS e AQLQ;

$\mathrm{H}_{15}$ : Correlação entre EQ-5D/VAS e ACQ;

$\mathrm{H}_{16}$ : Correlação entre EQ-5D/VAS e CCQ.

$\mathrm{H}_{17}$ : Correlação entre EQ-5D/VAS e AIMS2-SF.

Os dados foram analisados com o programa IBM SPSS Statistics, versão 20. Para todas as análises estatísticas, o nível máximo de significância foi fixado em $5 \%$.

\section{RESULTADOS}

\section{Adaptação semântica e linguística}

A versão portuguesa do EQ-5D foi criada em 1997 pelo CEISUC tendo-se seguido o processo de tradução/retroversão clássico. ${ }^{35-37}$ Mais tarde, num processo de harmonização entre as versões em língua de origem latina surgiram alguns aspetos que exigiram solução. Foi o caso do terceiro nível de resposta da pergunta sobre mobilidade. Inicialmente, a frase 'I am confined to bed' tinha sido traduzida por 'Eu estou acamado(a)' com uma carga demasiado pesada, tendo em conta a intensidade prevista pelos autores na versão original. Foi decidido substituir por 'Tenho de estar na cama'.

Nas opções de resposta da dimensão 'atividades habituais' os autores da versão original questionaram se o verbo 'desempenhar' deveria ou não ser substituído pelo verbo 'realizar' de modo a ser mais bem compreendido pelos portugueses. Considerou-se que, apesar do verbo utilizado na versão espanhola ser este, seria mais correto manter a proposta inicial portuguesa.

O terceiro aspeto teve a ver com a dimensão 'dor/mal-estar' e com a colocação dos adjetivos 'moderados' e 'extremos' no fim das frases para garantir que diziam respeito quer às dores quer ao mal-estar. Tratou-se efetivamente de 
Tabela 3 - Distribuição dos dados sociodemográficos e clínicos.

\begin{tabular}{|c|c|c|c|c|c|c|c|}
\hline \multirow{2}{*}{ Variável } & \multirow{2}{*}{ Valores } & \multicolumn{2}{|c|}{ Amostra 1} & \multicolumn{2}{|c|}{ Amostra 2} & \multicolumn{2}{|c|}{ Amostra 3} \\
\hline & & $\mathrm{N}$ & $\%$ & $\mathrm{~N}$ & $\%$ & $\mathrm{~N}$ & $\%$ \\
\hline Tamanho & & 1500 & 100,0 & 150 & 100,0 & 643 & 100,0 \\
\hline Género & $\begin{array}{l}\text { Feminino } \\
\text { Masculino }\end{array}$ & $\begin{array}{l}789 \\
711\end{array}$ & $\begin{array}{l}52,6 \\
47,4\end{array}$ & $\begin{array}{l}78 \\
72\end{array}$ & $\begin{array}{l}52,0 \\
48,0\end{array}$ & $\begin{array}{l}377 \\
265\end{array}$ & $\begin{array}{l}58,7 \\
41,3\end{array}$ \\
\hline Idade & $\begin{array}{l}18-29 \text { anos } \\
30-49 \text { anos } \\
50-69 \text { anos } \\
70 \text { ou + anos } \\
\text { Mínimo } \\
\text { Máximo } \\
\text { Média } \\
\text { Desvio padrão }\end{array}$ & $\begin{array}{r}346 \\
494 \\
388 \\
272 \\
18 \\
91 \\
48,0 \\
18,8\end{array}$ & $\begin{array}{l}23,1 \\
32,9 \\
25,9 \\
18,1\end{array}$ & $\begin{array}{r}39 \\
44 \\
50 \\
17 \\
18 \\
85 \\
46,4 \\
17,7\end{array}$ & $\begin{array}{l}26,0 \\
29,3 \\
33,3 \\
11,3\end{array}$ & $\begin{array}{r}12 \\
100 \\
206 \\
320 \\
18 \\
92 \\
64,9 \\
15,5\end{array}$ & $\begin{array}{r}1,9 \\
15,7 \\
32,3 \\
50,2\end{array}$ \\
\hline Situação familiar & $\begin{array}{l}\text { Solteiro/a } \\
\text { Casado/a ou em união de facto } \\
\text { Divorciado/a ou separado/a } \\
\text { Viúvo/a }\end{array}$ & $\begin{array}{l}375 \\
894 \\
100 \\
123\end{array}$ & $\begin{array}{r}25,2 \\
59,9 \\
6,7 \\
8,2\end{array}$ & $\begin{array}{r}41 \\
91 \\
9 \\
8\end{array}$ & $\begin{array}{r}27,5 \\
61,1 \\
6,0 \\
5,4\end{array}$ & $\begin{array}{r}52 \\
419 \\
40 \\
132\end{array}$ & $\begin{array}{r}8,1 \\
65,2 \\
6,2 \\
20,5\end{array}$ \\
\hline Nível de escolaridade & $\begin{array}{l}\leq 6 \text { anos } \\
7-12 \text { anos } \\
>12 \text { anos }\end{array}$ & $\begin{array}{l}469 \\
682 \\
337\end{array}$ & $\begin{array}{l}31,5 \\
45,8 \\
22,6\end{array}$ & $\begin{array}{l}39 \\
69 \\
41\end{array}$ & $\begin{array}{l}26,2 \\
46,3 \\
27,5\end{array}$ & $\begin{array}{r}469 \\
111 \\
63\end{array}$ & $\begin{array}{r}72,9 \\
17,3 \\
9,8\end{array}$ \\
\hline Situação perante o trabalho & $\begin{array}{l}\text { Empregado/a } \\
\text { Desempregado/a } \\
\text { Reformado/a ou aposentado/a } \\
\text { Estudante } \\
\text { Doméstico/a } \\
\text { Outra situação }\end{array}$ & $\begin{array}{r}725 \\
176 \\
380 \\
127 \\
83 \\
1\end{array}$ & $\begin{array}{r}48,6 \\
11,7 \\
25,5 \\
8,5 \\
5,6 \\
0,1\end{array}$ & $\begin{array}{r}60 \\
29 \\
39 \\
14 \\
7 \\
1\end{array}$ & $\begin{array}{r}40,0 \\
19,3 \\
26,0 \\
9,3 \\
4,7 \\
0,7\end{array}$ & $\begin{array}{r}179 \\
15 \\
416 \\
0 \\
31 \\
0\end{array}$ & $\begin{array}{r}27,9 \\
2,3 \\
64,9 \\
0,0 \\
4,8 \\
0,0\end{array}$ \\
\hline Agregado familiar & $\begin{array}{l}1 \text { a } 2 \text { elementos } \\
3 \mathrm{ou}+\text { elementos }\end{array}$ & $\begin{array}{l}642 \\
840\end{array}$ & $\begin{array}{l}43,3 \\
56,7\end{array}$ & $\begin{array}{l}61 \\
89\end{array}$ & $\begin{array}{l}40,7 \\
59,3\end{array}$ & $\begin{array}{r}587 \\
56\end{array}$ & $\begin{array}{r}91,3 \\
8,7\end{array}$ \\
\hline Com doença & $\begin{array}{l}\text { Sim } \\
\text { Não }\end{array}$ & $\begin{array}{l}594 \\
894\end{array}$ & $\begin{array}{l}39,9 \\
60,1\end{array}$ & $\begin{array}{l}58 \\
91\end{array}$ & $\begin{array}{l}38,9 \\
61,1\end{array}$ & $\begin{array}{r}643 \\
0\end{array}$ & $\begin{array}{r}100,0 \\
0,0\end{array}$ \\
\hline
\end{tabular}

uma opção de tradução que foi imediatamente alterada.

Também nas frases da introdução à escala visual analógica era inicialmente utilizada a palavra 'quão'. Isto ocorria nas frases 'Para ajudar as pessoas a classificarem quão bom ou mau é o seu estado de saúde hoje' e 'Gostaríamos que indicasse nesta escala quão bom ou mau é, na sua opinião, o seu estado de saúde hoje'. No processo de harmonização foi decidido que esta palavra, apesar de existir em português, não é comummente utilizada e poderia criar problemas de compreensão a alguns respondentes. Assim, estas frases foram reescritas sem a utilização desta palavra.

Desta revisão foi elaborada, em 1998, a versão portuguesa do EQ-5D que, desde então, passou a ser utilizada em Portugal, estando disponível, a pedido, a partir do CEISUC.

\section{Amostra}

Os dados sociodemográficos dos indivíduos das três amostras estão representados na Tabela 3.

Em todas as amostras encontrámos uma ligeira predominância do sexo feminino e de indivíduos casados ou em união de facto. A amostra 3 apresenta, como seria de esperar dadas as patologias, uma população mais idosa, com um nível de escolaridade mais baixo, reformados e a viver sós ou com o cônjuge.

\section{Aceitabilidade e distribuição}

A Tabela 4 apresenta a distribuição das respostas às cinco dimensões do EQ-5D e da EQ-VAS em cada uma das amostras.

No conjunto das três amostras, 2293 indivíduos completaram o questionário $E Q-5 D$, sem qualquer dado em falta nas três primeiras dimensões, $1(0,1 \%)$ dado em falta na dimensão dor/mal-estar e $7(0,5 \%)$ dados em falta na dimensão ansiedade/depressão, o que confirma a hipótese $H_{1}$. Não houve qualquer problema de compreensão ou dificuldade na resposta às perguntas. Em relação à EQ-VAS, apenas 25 pessoas $(1,7 \%)$ não responderam a esta pergunta na amostra 1, $5(3,3 \%)$ na amostra 2 e 52 $(8,1 \%)$ na amostra 3 , confirmando-se a hipótese $H_{2}$.

Entre as cinco dimensões do EQ-5D, a proporção de ter um qualquer problema foi maior para a dor/mal-estar e para a ansiedade/depressão. A média do EQ-VAS foi de 74,9 para as amostras 1 e 2 , e de 58,6 para a amostra $3 \mathrm{com}$ indivíduos doentes.

Confirmando-se a hipótese $\mathrm{H}_{3}$, encontrou-se evidência de efeito de teto, i.e., poucos indivíduos se posicionaram 
Tabela 4 - Distribuição das respostas das dimensões EQ-5D da ERQ-VAS nas três amostras.

\begin{tabular}{|c|c|c|c|c|c|c|c|}
\hline \multirow{2}{*}{ EQ-5D } & & \multicolumn{2}{|c|}{ Amostra 1} & \multicolumn{2}{|c|}{ Amostra 2} & \multicolumn{2}{|c|}{ Amostra 3} \\
\hline & & $\mathrm{N}$ & $\%$ & $\mathrm{~N}$ & $\%$ & $\mathrm{~N}$ & $\%$ \\
\hline \multirow{3}{*}{ Mobilidade } & Sem problemas & 1249 & 83,3 & 129 & 86,0 & 405 & 63,0 \\
\hline & Problemas moderados & 244 & 16,3 & 21 & 14,0 & 238 & 37,0 \\
\hline & Problemas extremos & 7 & 0,5 & 0 & 0,0 & 0 & 0,0 \\
\hline \multirow{3}{*}{ Cuidados pessoais } & Sem problemas & 1.428 & 95,2 & 145 & 96,7 & 561 & 87,2 \\
\hline & Problemas moderados & 66 & 4,4 & 5 & 3,3 & 73 & 11,4 \\
\hline & Problemas extremos & 6 & 0,4 & 0 & 0,0 & 9 & 1,4 \\
\hline \multirow{3}{*}{ Atividades habituais } & Sem problemas & 1.255 & 83,7 & 125 & 83.3 & 402 & 62,5 \\
\hline & Problemas moderados & 209 & 13,9 & 23 & 15,3 & 235 & 36,5 \\
\hline & Problemas extremos & 36 & 2,4 & 2 & 1,3 & 6 & 0,9 \\
\hline \multirow{3}{*}{ Dor/mal-estar } & Sem problemas & 830 & 55,4 & 80 & 53,3 & 256 & 39,8 \\
\hline & Problemas moderados & 599 & 40,0 & 66 & 44,0 & 350 & 54,4 \\
\hline & Problemas extremos & 70 & 4,7 & 4 & 2,7 & 37 & 5,8 \\
\hline \multirow{3}{*}{ Ansiedade/depressão } & Sem problemas & 980 & 65,6 & 102 & 68,0 & 283 & 44,0 \\
\hline & Problemas moderados & 449 & 30,1 & 43 & 28,7 & 330 & 51,3 \\
\hline & Problemas extremos & 64 & 4,3 & 5 & 3,3 & 30 & 4,7 \\
\hline \multirow{11}{*}{ EQ-VAS } & Percentil 5 & 34,8 & & 40,0 & & 30,0 & \\
\hline & Percentil 10 & 50,0 & & 50,0 & & 37,0 & \\
\hline & Percentil 25 & 60,0 & & 60,0 & & 50,0 & \\
\hline & Percentil 50 & 80,0 & & 80,0 & & 59,0 & \\
\hline & Percentil 75 & 90,0 & & 90,0 & & 70,0 & \\
\hline & Percentil 90 & 99,0 & & 100,0 & & 80,8 & \\
\hline & Percentil 95 & 100,0 & & 100,0 & & 90,0 & \\
\hline & Mínimo & 0,0 & & 5,0 & & 20,0 & \\
\hline & Máximo & 100,0 & & 100,0 & & 100,0 & \\
\hline & Média & 74,9 & & 74,9 & & 58,6 & \\
\hline & Desvio padrão & 21,2 & & 20,3 & & 17,0 & \\
\hline
\end{tabular}

Tabela 5 - Distribuição das dimensões EQ-5D nas subamostras por patologia.

\begin{tabular}{|c|c|c|c|c|c|c|c|c|c|}
\hline \multirow[t]{2}{*}{ Dimensão EQ-5D } & & \multicolumn{2}{|c|}{$\begin{array}{c}\text { Cataratas }^{15} \\
(n=352)\end{array}$} & \multicolumn{2}{|c|}{$\begin{array}{c}\text { Asma }^{14} \\
(n=115)\end{array}$} & \multicolumn{2}{|c|}{$\begin{array}{l}\mathrm{DPOC}^{36} \\
(n=72)\end{array}$} & \multicolumn{2}{|c|}{$\begin{array}{c}\mathrm{AR}^{16} \\
(n=104)\end{array}$} \\
\hline & & $\mathbf{N}$ & $\%$ & $\mathbf{N}$ & $\%$ & $\mathbf{N}$ & $\%$ & $\mathbf{N}$ & $\%$ \\
\hline \multirow{3}{*}{ Mobilidade } & Sem problemas & 220 & 62,5 & 82 & 71,3 & 55 & 76,4 & 48 & 46,2 \\
\hline & Problemas moderados & 132 & 37,5 & 33 & 28,7 & 17 & 23,6 & 56 & 53,8 \\
\hline & Problemas extremos & 0 & 0,0 & 0 & 0,0 & 0 & 0,0 & 0 & 0,0 \\
\hline \multirow{3}{*}{ Cuidados pessoais } & Sem problemas & 316 & 89,1 & 109 & 94,8 & 68 & 94,4 & 68 & 65,4 \\
\hline & Problemas moderados & 32 & 9,1 & 6 & 5,2 & 3 & 4,2 & 32 & 30,8 \\
\hline & Problemas extremos & 4 & 1,1 & 0 & 0,0 & 1 & 1,4 & 4 & 3,8 \\
\hline \multirow{3}{*}{ Atividades habituais } & Sem problemas & 220 & 62,5 & 87 & 75,7 & 55 & 76,4 & 40 & 38,5 \\
\hline & Problemas moderados & 128 & 36,4 & 28 & 24,3 & 15 & 20,8 & 64 & 61,5 \\
\hline & Problemas extremos & 4 & 1,1 & 0 & 0,0 & 2 & 2,8 & 0 & 0,0 \\
\hline \multirow{3}{*}{ Dor/mal-estar } & Sem problemas & 108 & 30,7 & 76 & 66,1 & 56 & 77,8 & 16 & 15,4 \\
\hline & Problemas moderados & 216 & 61,4 & 38 & 33,0 & 16 & 22,2 & 80 & 76,9 \\
\hline & Problemas extremos & 28 & 8,0 & 1 & 0,9 & 0 & 0,0 & 8 & 7,7 \\
\hline \multirow{3}{*}{ Ansiedade/depressão } & Sem problemas & 136 & 38,6 & 71 & 61,7 & 52 & 72,2 & 24 & 23,1 \\
\hline & Problemas moderados & 192 & 54,5 & 43 & 37,4 & 19 & 26,4 & 76 & 73,1 \\
\hline & Problemas extremos & 24 & 6,8 & 1 & 0,9 & 1 & 1,4 & 4 & 3.8 \\
\hline
\end{tabular}

no nível 3 das cinco dimensões. De facto, nas amostras 1 e 2, as dimensões mobilidade, cuidados pessoais e atividades habituais apresentaram sempre valores superiores a $83 \%$ no nível 1 (sem limitação). Além disso, nas dimensões dor/mal-estar e ansiedade/depressão, mais de metade dos indivíduos escolheram o nível 1. Fenómeno semelhante ocorreu na amostra 3 em que os pontos de corte foram, naturalmente, mas baixos mas fixaram-se, respetivamente, em $62 \%$ e $40 \%$, no entanto ainda multo altos. Assim, olhando com mais detalhe para as subamostras por patologia da amostra 3, pode ver-se na Tabela 5 que este efeito de teto ainda é mais notório.

Da Tabela anterior é evidente para as cataratas, a asma e a DPOC que as distribuições das dimensões associa- 
das à mobilidade, aos cuidados pessoais e às atividades habituais têm a moda no primeiro nível, o que revela um acentuado efeito de teto, tratando-se de doentes, na sua maioria, idosos com uma doença limitativa do desempenho das suas atividades habituais. O mesmo acontece para as dimensões dor/mal-estar e ansiedade/depressão nos doentes com asma e DPOC e com a dimensão cuidados pessoais dos doentes com AR. Já nas dimensões dor/mal-estar e ansiedade/depressão dos doentes com cataratas e nas restantes quatro dimensões dos doentes com AR, a moda localiza-se no segundo nível.

\section{Fiabilidade}

A Tabela 6 apresenta três indicadores que demonstram a fiabilidade deste instrumento de medição: percentagem de concordância, coeficiente de correlação de Spearman e coeficiente k de Cohen.

Os valores encontrados indicam valores muito bons de correlação e valores de $\mathrm{k}$ moderados (cuidados pessoais $\mathrm{e}$ dor/mal-estar) a bons (restantes dimensões).

O valor $\alpha$ de Cronbach para o EQ-5D foi de 0,716 indicando uma coerência interna aceitável. Por outro lado, o coeficiente intraclasse ICC para a EQ-VAS entre os dois períodos foi elevado $(0,862$ com intervalo de confiança de $0,808$ a 0,901$)$, assim como o coeficiente de correlação de Pearson $(0,768 ; p<0,001)$.

\section{Validade de construção}

Para responder à questão da validade de construção, relacionando as dimensões do EQ-5D com as do SF-36v2, e às primeiras três hipóteses formuladas nesta secção, a Tabela 7 apresenta, para cada nível sem/com problemas de cada uma das dimensões do EQ-5D, o valor da mediana das pontuações das várias dimensões SF-36v2 e o respetivo indicador de significância estatística.

Assim, conforme indicado na hipótese $\mathrm{H}_{4}$, as distribuições dos indivíduos que reportaram problemas moderados ou extremos em cada dimensão EQ-5D apresentaram sempre uma mediana significativamente mais baixa dos que não tinham reportando problemas, tal como já referido. ${ }^{38}$ Também em relação à dimensão ansiedade/depressão a diferença em valores de mediana para a escala SM do SF-36v2 (32,0 pontos) e FS (25,0 pontos) são maiores do que a diferença na FF (15,0 pontos), como indicado na hipótese $\mathrm{H}_{5}$ e já também defendido. ${ }^{39} \mathrm{~A}$ diferença de pontos entre as medianas da dimensão DR do SF-36v2 entre os que no EQ-5D referem não ter dores e os que referem problemas com a dor é de 39,0 pontos (hipóteses $\mathrm{H}_{6}$ ), conforme anteriormente demonstrado. ${ }^{40}$

Para responder à hipótese $\mathrm{H}_{7}$, a Tabela 8 indica a relação entre as respostas às dimensões do EQ-5D, a idade e a presença de doença.

Tabela 6 - Fiabilidade do EQ-5D.

\begin{tabular}{lccc}
\hline Dimensão EQ-5D & Concordância (\%) & Correlação (a) & k de Cohen \\
\hline Mobilidade & 91,9 & 0,677 & 0,647 \\
Cuidados pessoais & 97,3 & 0,586 & 0,586 \\
Atividades habituais & 89,3 & 0,661 & 0,308 \\
Dor/mal-estar & 77,3 & 0,633 & 0,555 \\
Ansiedade/depressão & 85,0 & 0,661 & 0,633
\end{tabular}

Tabela 7 - Medianas do SF-36v2 para as várias dimensões EQ-5D.

\begin{tabular}{|c|c|c|c|c|c|c|c|c|c|}
\hline Dimensão EQ-5D & $\mathbf{N}$ & $\mathbf{F F}$ & DF & DR & SG & DE & VT & FS & SM \\
\hline Mobilidade & & $* *$ & $* *$ & ** & $* *$ & ** & $* *$ & ** & ** \\
\hline Sem problemas & 1.249 & 95,0 & 93,7 & 74,0 & 62,0 & 91,7 & 70,0 & 87,5 & 80,0 \\
\hline Com problemas & 251 & 45,0 & 50,0 & 51,0 & 44,4 & 58,3 & 45,0 & 62,5 & 56,0 \\
\hline Cuidados pessoais & & ** & $* *$ & $* *$ & $* *$ & ** & $* *$ & ** & ** \\
\hline Sem problemas & 1.428 & 90,0 & 93,7 & 72,0 & 62,0 & 91,7 & 70,0 & 87,5 & 80,0 \\
\hline Com problemas & 72 & 25,0 & 31,3 & 31,0 & 40,0 & 41,7 & 35,0 & 50,0 & 52,0 \\
\hline Atividades habituais & & ** & ** & $* *$ & ** & ** & $* *$ & ** & ** \\
\hline Sem problemas & 1.255 & 95,0 & 93,7 & 72,0 & 62,0 & 91,7 & 70,0 & 87,5 & 84,0 \\
\hline Com problemas & 245 & 45,0 & 50,0 & 42,0 & 40,0 & 58,3 & 40,0 & 62,5 & 55,0 \\
\hline Dor/mal-estar & & ** & ** & $* *$ & ** & ** & $* *$ & ** & ** \\
\hline Sem problemas & 830 & 95,0 & 100,0 & 100,0 & 67,0 & 100,0 & 75,0 & 100,0 & 84,0 \\
\hline Com problemas & 669 & 75,0 & 68,7 & 61,0 & 50,0 & 75,0 & 50,0 & 75,0 & 68,0 \\
\hline Ansiedade/depressão & & ** & ** & ** & ** & ** & ** & ** & ** \\
\hline Sem problemas & 980 & 95,0 & 100,0 & 74,0 & 65,0 & 100,0 & 71,7 & 100,0 & 88,0 \\
\hline Com problemas & 513 & 80,0 & 75,0 & 62,0 & 50,0 & 66,7 & 50,0 & 75,0 & 56,0 \\
\hline
\end{tabular}

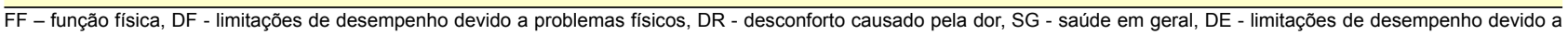
problemas emocionais, VT - vitalidade, FS - função social, SM - saúde mental. ** $p<0,001$ 
Como se vê, os mais idosos e os mais doentes são os que significativamente reportam mais problemas em todas as dimensões do EQ-5D.

A Tabela 9 apresenta os valores médios da EQ-VAS para cada um dos níveis das variáveis que permitem testar as hipóteses $\mathrm{H}_{8}$ a $\mathrm{H}_{11}$.

A EQ-VAS foi analisada, primeiramente, face à autoavaliação do estado de saúde (pergunta 1 do SF-36v2) e ao grupo etário. Como se observa neste quadro, a EQ-VAS está fortemente relacionada com a autoavaliação da saúde, pois a diferença entre as médias de cada nível de saúde é significativa $(p<0,001$, com exceção do salto entre os níveis 'ótima' e 'muito boa'), confirmando-se a hipótese $\mathrm{H}_{8}$, também defendida por outros autores. ${ }^{41,42}$

Por outro lado (hipótese $\mathrm{H}_{9}$ ), em relação à idade, verifica-se que o EQ-VAS decresce significativamente à medida que a idade avança $(p<0,001$ para todas os saltos entre os grupos etários, exceto entre os grupos 18-29 e 30-49 anos em que $p<0,01$ ), confirmando outros resultados. ${ }^{42,43}$

Também os indivíduos doentes apresentaram avaliações globais do seu estado de saúde piores do que os saudáveis (hipótese $\mathrm{H}_{10}$ ), o mesmo acontecendo com mulheres relativamente aos homens (hipótese $\mathrm{H}_{11}$ ).

\section{Validade de critério}

Para testar este tipo de validade, compararam-se finalmente os valores recolhidos pelo EQ-5D, primeiro com os obtidos pelo SF-6D e, de seguida, com os obtidos por outros instrumentos específicos de medição. A Tabela 10 reporta estes resultados.

Em termos de comparação entre instrumentos de medição baseados em preferências (hipótese $\mathrm{H}_{12}$ ), e apesar de se tratar de medidas com sistemas descritos diferentes, compararam-se os resultados do EQ-5D e da EQ-VAS com as dimensões do SF-6D. Encontrámos correlações de intensidade moderada, em sintonia com outros estudos. ${ }^{44-46}$

Em relação à subamostra de doentes com cataratas, compararam-se os índices EQ-5D com as áreas do Catquest (hipótese $\mathrm{H}_{13}$ ). Demonstrou-se, como esperado, a existência de correlações moderadas e inversas entre a medida de utilidade e o nível de atividade, os sintomas de cataratas e a opinião geral do Catquest. ${ }^{17}$ Ainda de realçar uma correlação também moderada e inversa entre a EQ-VAS e a incapacidade, o que pode significar que a meIhoria em alguma destas áreas poderá levar a um aumento da utilidade do estado de saúde dos doentes e, assim a uma melhoria da QdVRS.

Ao analisar a concordância entre o EQ-5D e os resultados dos instrumentos específicos para a asma, verificámos que os domínios do $A Q L Q(S)$ e os respetivos valores globais se encontram direta e moderada/fortemente correlacionados com as medidas de utilidade (hipótese $\mathrm{H}_{14}$ ),

Tabela 8 - Relações entre as respostas ao EQ-5D, idade e doença, N (\%).

\section{Dimensões EQ-5D com problemas}

\begin{tabular}{|c|c|c|c|c|c|}
\hline Variável & Mobilidade & Cuidados pessoais & Atividades habituais & Dor/ mal-estar & Ansiedade/depressão \\
\hline Idade & ** & ** & ** & ** & ** \\
\hline$<65$ anos & $115(10,3 \%)$ & $38(3,4 \%)$ & $121(10,8 \%)$ & $409(36,5 \%)$ & $361(32,3 \%)$ \\
\hline$\geq 65$ anos & $134(36,7 \%)$ & $33(9,0 \%)$ & $121(33,2 \%)$ & $250(68,7 \%)$ & $146(40,6 \%)$ \\
\hline Doença & ** & $\star *$ & ** & ** & ** \\
\hline Não & $63(7,0 \%)$ & $11(1,2 \%)$ & $50(5,6 \%)$ & $273(30,5 \%)$ & $225(25,3 \%)$ \\
\hline Sim & $185(31,1 \%)$ & $61(10,3 \%)$ & $192(32,3 \%)$ & $385(64,9 \%)$ & $282(47,6 \%)$ \\
\hline
\end{tabular}

Tabela 9 - Relação entre a EQ-VAS, a autoavaliação da saúde e varáveis de contexto.

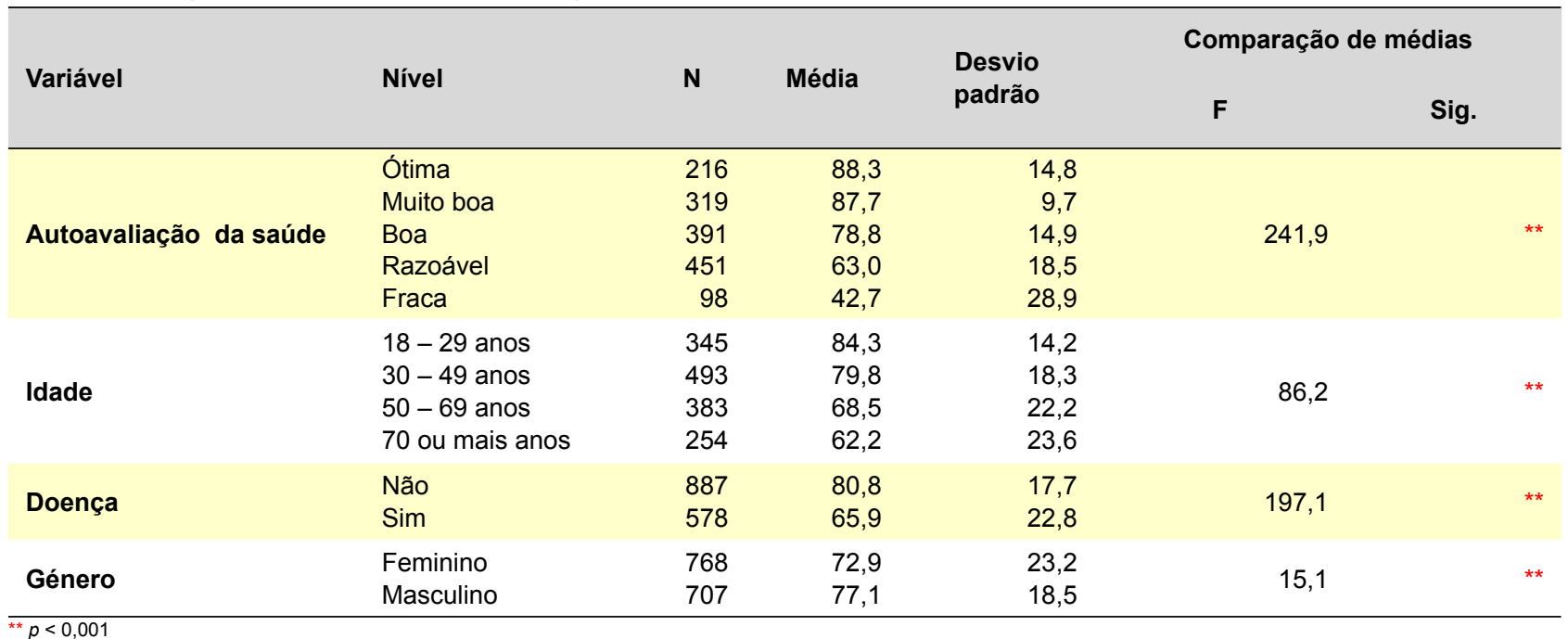


Tabela 10 - Correlação entre SF-6D e EQ-5D.

\begin{tabular}{|c|c|c|c|}
\hline Instrumento de medição & Dimensões & EQ-5D & EQ-VAS \\
\hline SF-6D & $\begin{array}{l}\text { Função física } \\
\text { Limitação de desempenho } \\
\text { Função social } \\
\text { Dor } \\
\text { Saúde mental } \\
\text { Vitalidade }\end{array}$ & $\begin{array}{l}-0,520^{* *} \\
-0,412^{* *} \\
-0,419^{* *} \\
-0,509^{* *} \\
-0,480^{* *} \\
-0,442^{* *}\end{array}$ & $\begin{array}{l}-0,486^{* *} \\
-0,449^{\star *} \\
-0,428^{* *} \\
-0,607^{\star *} \\
-0,541^{* *} \\
-0,411^{* *}\end{array}$ \\
\hline Catquest & $\begin{array}{l}\text { Incapacidade } \\
\text { Sintomas de cataratas } \\
\text { Nível de atividade } \\
\text { Opinião geral } \\
\text { Condução }\end{array}$ & $\begin{array}{l}-0,07 \\
-0,13^{*} \\
-0,29^{* * *} \\
-0,29^{* * *} \\
0,10\end{array}$ & $\begin{array}{l}-0,20^{* *} \\
-0,31^{* * *} \\
-0,23^{* * *} \\
-0,33^{* * *} \\
0,08\end{array}$ \\
\hline AQLQ(S) & $\begin{array}{l}\text { Limitações de atividade } \\
\text { Sintomas } \\
\text { Função emocional } \\
\text { Exposição ambiental } \\
\text { Valor global }\end{array}$ & $\begin{array}{l}0,50^{* * *} \\
0,56^{* * *} \\
0,33^{* * *} \\
0,31^{* * *} \\
0,53^{* * *}\end{array}$ & $\begin{array}{l}0,55^{* * *} \\
0,53^{* * *} \\
0,35^{* * *} \\
0,37^{* * *} \\
0,55^{* * *}\end{array}$ \\
\hline$A C Q$ & Valor global & $-0,51^{* * *}$ & $-0,51^{* * *}$ \\
\hline CCQ & $\begin{array}{l}\text { Sintomas } \\
\text { Estado funcional } \\
\text { Estado mental } \\
\text { Valor global }\end{array}$ & $\begin{array}{l}-0,278^{*} \\
-0,527^{* *} \\
-0,059 \\
-0,358^{* *}\end{array}$ & $\begin{array}{l}-0,362^{* *} \\
-0,523^{* *} \\
-0,304^{* *} \\
-0,540^{* *}\end{array}$ \\
\hline AIMS2-SF & $\begin{array}{l}\text { Física } \\
\text { Sintomas } \\
\text { Afeto } \\
\text { Interação social } \\
\text { Desempenho }\end{array}$ & $\begin{array}{l}-0,01 \\
-0,93^{* *} \\
-0,40^{* *} \\
-0,41^{* *} \\
-0,05\end{array}$ & $\begin{array}{l}0,07 \\
-0,76^{\star *} \\
-0,29 \\
-0,59^{\star *} \\
0,14\end{array}$ \\
\hline
\end{tabular}

${ }^{*} p<0,05{ }^{* \star} p<0,01{ }^{* * *} p<0,001$

podendo significar que uma melhoria em alguma das áreas levará também a um aumento da utilidade. Por outro lado, verifica-se a existência de correlações fortes e inversas entre o valor global do ACQ e as medidas do EQ-5D (hipótese $\mathrm{H}_{15}$ ).

No que respeita a DPOC, a EQ-VAS apareceu correlacionada com todas as dimensões do CCQ e a parte descritiva do EQ-5D apresentou uma boa correlação com o valor global (hipótese $\mathrm{H}_{16}$ ).

Por fim, analisando os resultados da aplicação do instrumento específico de medição da qualidade de vida em doentes com AR, demonstrou-se a existência de correlações fortes e inversas entre as medidas de utilidade e as dimensões relacionadas com os sintomas, o afeto e a interação social do AIMS2-SF (hipótese $\mathrm{H}_{17}$ ).

\section{CONCLUSÃO}

Cada vez mais a medição de resultados em saúde é considerada vital para a monitorização da prática clínica, em especial quando os ganhos associados a tratamentos distintos são considerados marginais. Um dos instrumentos de medição utilizados para medir estes resultados em saúde é o EQ-5D. Aplicável a um amplo conjunto de condições de saúde e de tratamentos, o EQ-5D gera não só um perfil de saúde, mas também um índice que exprime a QdVRS dos indivíduos que respondem.

Os resultados obtidos pelo EQ-5D poderão ser utilizados no planeamento e alocação dos recursos no sector da saúde, tanto ao nível nacional, como regional e ainda por doença e serem incorporados no Plano Nacional de Saúde, à semelhança do que já se faz em outros países. Assim, existe uma clara aplicação prática dos resultados pelas autoridades de saúde, nacionais e/ou regionais.

Os valores de utilidade dos estados de saúde da população portuguesa poderão também ser utilizados nas avaliações económicas de tecnologia de saúde, tanto ao nível da avaliação económica de medicamentos, como de diferentes programas de saúde (em situações de escolha entre diferentes alternativas, deverá ser escolhida a alternativa que permitir atingir o estado de saúde com maior utilidade). No entanto, qualquer instrumento de medição, antes de ser implementado, necessita ter propriedades aceitáveis. Com este trabalho podemos afirmar as seguintes conclusões gerais relativamente à versão portuguesa do EQ-5D:

- A tradução e a adaptação cultural foram feitas com base na melhor prática defendida em termos científicos;

- Apresentou uma ótima aceitabilidade por parte dos respondentes com poucos dados omissos e a presença do esperado efeito de teto;

- A fiabilidade teste-reteste foi confirmada e considerada elevada e a coerência interna foi considerada aceitável;

- A validade de construção foi verificada através de hipóteses definidas a priori e da sua confirmação;

- A validade de critério foi testada com êxito através da análise do comportamento do EQ-5D e da EQ-VAS em relação ao outro instrumento baseado em preferências 
SF-6D e, apesar de se tratar de um instrumento genérico de medição em saúde, em relação às dimensões de instrumentos específicos para as cataratas, a asma, a DPOC e a AR.

Deste modo, podemos afirmar que a versão portuguesa do EQ-5D tem uma boa aceitabilidade e validade na medição do estado de saúde.

\section{AGRADECIMENTOS}

Os autores agradecem a Maria do Céu Machado, ex-Alta Comissária para a Saúde pelo apoio prestado. Agradecem ainda às equipas médicas e de enfermagem do Centro Hospitalar do Barlavento Algarvio e do Hospital Central de
Faro que recolheram os dados das cataratas, aos médicos pneumologistas e às técnicas de cardiopneumologia do Hospital Central de Faro que recolheram os dados dos doentes com asma e DPOC, e à LPCDR e aos médicos reumatologistas que permitiram a recolha de dados da AR.

\section{CONFLITOS DE INTERESSE}

Nada a declarar

\section{FONTES DE FINANCIAMENTO}

O Alto Comissariado para a Saúde e os Laboratórios Pfizer concederam o apoio financeiro que permitiu a recoIha dos dados das amostras 1 e 2 .

\section{REFERÊNCIAS}

1. Brooks R. EuroQoL: the current state of play. Health Policy. 1996;37:5372.

2. EuroQoL Group. EuroQol - A new facility for the measurement of healthrelated quality of life. Health Policy. 1990;16:199-208.

3. Torrance GW. Measurement of health state utilities for economical appraisal: a review. J Health Econ. 1986; 5:1-30.

4. Nord E. EuroQoL: health-related quality of life measurement. Valuation of health states by the general public in Norway. Health Policy. 1991;18:25-36

5. Dolan P. Modeling valuations for EuroQol health states. Med Care. 1997;35:1095-108.

6. Dolan P, Gutex C, Kind P, Williams A. A social tariff for EuroQoL: results from a U.K. general population survey. Discussion Paper 138. York: Centre for Health Economics; 1995.

7. Williams A. The measurement and valuation of health: a chronicle. Discussion Paper 136. York: Centre for Health Economics; 1995.

8. Williams A. The role of the EuroQoL instrument in QALY calculations. Discussion Paper 130. York: Centre for Health Economics; 1995.

9. Chevalier J, de Pouvourville G. Valuing EQ-5D using Time Trade-Off in France. Eur J Health Econ. 2013;14:57-66.

10. Golicki D, Jakubczyk M, Niewada M, Wrona W, Busschbach J. Valuation of EQ-5D health states in Poland: first TTO-based social value in Central and Eastern Europe. Value Health. 2010;13:289-97.

11. Rabin R, Charro F, Szende A. Introduction. In: Szende A, Oppe M, Devlin $N$, editors. EQ-5D value sets - inventory, comparative review and user guide. Heidelberg: EuroQol Group Monographs Springer; 2007.

12. Shaw J, Johnson J, Coons S. US valuation of the EQ-5D health states: development and testing of the D1 model. Med Care. 2005;43:203-20.

13. Szende A, Williams A. Measuring self-reported population health: an international perspective based on EQ-5D. Budapest: SpringMed Publishing; 2004.

14. Ferreira LN, Ferreira PL, Pereira LN, Oppe M. The valuation of the EQ-5D in Portugal. Qual Life Res. (in press). 2013. DOI 10.1007/ s11136-013-0448-z.

15. Ferreira LN, Ferreira PL, Pereira LN, Oppe M. EQ-5D Portuguese population norms. Qual Life Res. (in press). 2013. DOI 10.1007/s11136-0130488-4

16. Ferreira L, Brito U, Ferreira P. Quality of life in asthma patients. Rev Port Pneumol. 2010;16:23-55

17. Ferreira L, Ferreira P. Qualidade de vida em doentes com cataratas. Oftalmologia. 2008;32:159-75.

18. Ferreira L, Ferreira P, Baleiro R. Qualidade de vida em doentes com artrite reumatóide. Acta Reumatol Port. 2008;33:341-2.

19. Landis JR, Koch GG. The measurement observer agreement for categorical data. Biometrics. 1977;33:159-74.

20. Terwee CB, Bot SD, de Boer MR, van der Windt DA, Knol DL, Dekker J, et al. Quality criteria we proposed for measurement properties of health status questionnaires. J Clin Epidemiol. 2007:60:34-42.

21. Ferreira PL. Criação da versão portuguesa do MOS SF-36. Parte I Adaptação cultural e linguística. Acta Med Port. 2000;13:55-66.

22. Ferreira PL. Criação da versão portuguesa do MOS SF-36. Parte II Testes de validação. Acta Med Port. 2000;13:119-27.

23. Ware JE, Sherbourne CD. The MOS 36-item Short-Form Health Sur- vey (SF-36). Conceptual framework and item selection. Med Care. 1992;30:473-83

24. Ware JE, Snow KK, Kosinski M, Gandek B. SF-36 Health survey manual and interpretation guide. Boston: The Health Institute; 1993.

25. Brazier J, Roberts J, Deverill M. The estimation of a preference-based measure of health from the SF-36. J Health Econ. 2002;21:271-92.

26. Ferreira L, Ferreira P, Pereira L, Brazier J, Rowen D. A Portuguese value set for the SF-6D. Value Health. 2010;13:624-30.

27. Ferreira L, Ferreira PL, Gonçalves S. Ganhos em saúde em doentes com cataratas. Notas Econ. 2006;23:35-53.

28. Lundström M, Roos $P$, Jensen $S$, Fregell G. Catquest questionnaire for use in cataract surgery care: Description, validity and reliability. J Cataract Refract Surg. 1997;23:1226-36.

29. Ferreira PL, Mendonça C, Newparth N, Mata P, Juniper EF, Mear I. Quality of life in asthma: cultural adaptation of the Juniper's AQLQ to Portuguese. Qual Life Res. 1998;7:590.

30. Juniper E, Buist A, Cox F, Ferrie P, King D. Validation of a standardized version of the Asthma Quality of Life Questionnaire. Chest. 1999:115:1265-70.

31. Juniper E, O'Byrne P, Guyatt G; Ferrie P, Kind D. Development and validation of a questionnaire to measure asthma control. Eur Resp J. 1999;14:902-7.

32. Van der Molen, T, Willemse, B, Schokker, S, Ten Hacken, N, Postma DS, Juniper EF. Development, validity and responsiveness of the Clinical COPDD Questionnaire. Health Qual Life Outcomes. 2003;1:13.

33. Meenan R, Gertman M, Mason J. Measuring health status in arthritis. The arthritis impact measurement scales. Arthritis Rheum. 1980;23:14652.

34. Guillemin F, Coste J, Pouchot J, Ghezail M., Bregeon C., Sany J. The AIMS2-SF: a short form of the arthritis impact measurement scales 2. Arthritis Rheum. 1997;40:1267-74

35. Acquadro C, Conway K, Giroudet C, Mear I. Linguistic validation manua for patient-reported outcomes (PRO) instruments. Lyon: Mapi Research Institute; 2004.

36. Guillemin F, Bombardier C, Beaton D. Cross-cultural adaptation of health-related quality of life measures: literature review and proposed guidelines. J Clin Epidemiol. 1993;46:1417-32.

37. Rabin R, Herdman M, Fox-Rushby J, Badia X. Exploring the results of translating the EQ-5D into 11 European languages. In: Brooks R, Rabin $\mathrm{R}$, de Charro $\mathrm{F}$, editors. The measurement and valuation of health status using EQ-5D: A European perspective. Dordrecht: Kluwer Academic Publishers; 2003

38. Ferreira L. Medição da utilidade dos estados de saúde da população portuguesa. Tese de Doutoramento em Economia. Coimbra: Faculdade de Economia da Universidade de Coimbra; 2009.

39. Brazier J, Jones N, Kind P. Testing the ability of the Euroqol and comparing it with the SF-46 health survey questionnaire. Quality Life Res. 1993;2:169-80.

40. Hurst NP, Kind P, Ruta D, Hunter M, Stubbings A. Measuring healthrelated quality of life in rheumatoid arthritis: validity, responsiveness and reliability of EuroQol (EQ-5D). Br J Rheumatol. 1997;36:551-9.

41. Badia X, Schiaffino A, Alonso J, Herdman M. Using the EuroQol 5-D in the Catalan general population: feasibility and construct validity. Quality 
Life Res. 1998;7:311-322.

42. Badia X, Herdman M, Schiaffino A. Determining correspondence between scores on the EQ-5D 'thermometer' and a 5-point categorical rating scale. Med Care. 1999;37:671-7.

43. Kind P, Dolan P, Gutex C, Williams A. Variations in population health status: results from a United Kingdom national questionnaire survey. BMJ. 1998;316:736-41.

44. Brazier J, Roberts J, Tsuchiya A, Busschbach J. A comparison of the
EQ-5D and SF-6D across seven patient groups. Health Econ. 2004;13:873-84.

45. Grieve R, Grishchenko M, Cairns J. SF-6D versus EQ-5D: reasons for differences in utility scores and impact on reported cost-utility. Eur $\mathrm{J}$ Health Econ. 2009;10:15-23.

46. Lamers L, Bouwmans C, van Straten A, Donker M, Hakkaart L. Comparison of EQ-5D and SF-6D utilities in mental health patients. Health Econ. 2006;15:1229-36. 


\section{Contributos para a Validação da Versão Portuguesa do EQ-5D \\ Acta Med Port 2013:26:664-675}

Publicado pela Acta Médica Portuguesa, a Revista Científica da Ordem dos Médicos

Av. Almirante Gago Coutinho, 151

1749-084 Lisboa, Portugal.

Tel: +351218428215

E-mail: submissao@actamedicaportuguesa.com

www.actamedicaportuguesa.com

ISSN:0870-399X | e-ISSN: 1646-0758

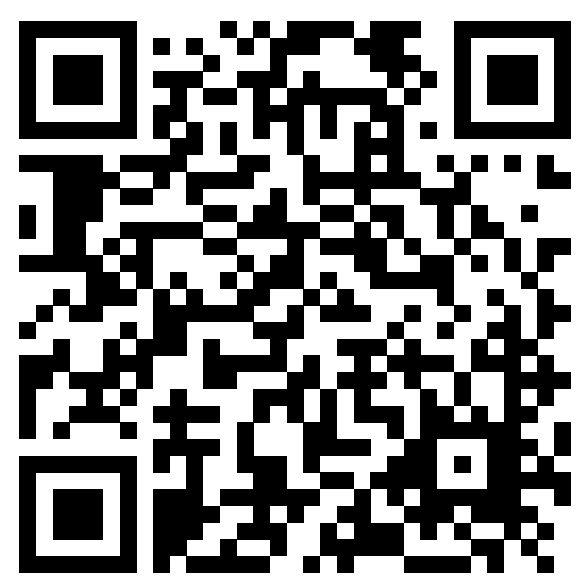

